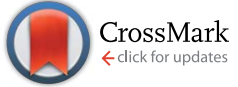

Cite this: RSC Adv., 2017, 7, 1318

Received 1st November 2016 Accepted 20th December 2016

DOI: 10.1039/c6ra26136a

www.rsc.org/advances

\section{A fluorescent carbon nitride nanofibrous hydrogel for selective sensing of $\mathrm{Cu}^{2+}$}

\author{
Zhijun Huang, Feng-Wen Yan* and Guoqing Yuan* \\ A carbon nitride nanofibrous hydrogel was fabricated by self-assembly/gelation of carbon nitride aqueous \\ solution derived from thermal evaporation of bulk graphitic carbon nitride. The structure, chemical \\ composition and optical properties of the as-prepared nanofibers were well investigated. The $\pi-\pi$ \\ interactions between nitrogen containing aromatic rings and hydrogen bonds between hydroxyl and \\ amino groups were the driving forces for the formation of nanofibers and the hydrogel. A fluorescent \\ nanofiber suspension can be obtained by dilution of the hydrogel with water. Due to the excellent \\ fluorescence properties and the high dispersion stability in water, the carbon nitride nanofibers can be \\ employed as a fluorescent probe for the selective sensing of $\mathrm{Cu}^{2+}$.
}

\section{Introduction}

Carbon materials have long been used in adsorption, separation, catalysis and electrochemistry. Heteroatom-doping is an effective method to improve the performance and expand the application of carbon based materials. ${ }^{1}$ By far, nitrogen is the most widely investigated heteroatom. ${ }^{2}$ Both the chemical reactivity and optical and electronic properties could be improved by nitrogen-doping. ${ }^{3,4}$

Graphitic carbon nitride $\left(\mathrm{g}-\mathrm{C}_{3} \mathrm{~N}_{4}\right)$, a nitrogen-rich carbon material, has received much interest due to its unusual optical and electronic properties and surprisingly high thermal and chemical stability. ${ }^{5,6}$ It is generally prepared by directly condensation of nitrogen and carbon containing precursors, such as $s$-triazine based compounds, ${ }^{7,8}$ urea $^{9}$ and guanidine salts. ${ }^{10}$ This in situ preparation technique provides $\mathrm{g}-\mathrm{C}_{3} \mathrm{~N}_{4}$ with a homogeneously nitrogen-doped carbon backbone. It is composed of tri-s-triazine units connected via trigonal nitrogen atoms. Over the past decade, $\mathrm{g}-\mathrm{C}_{3} \mathrm{~N}_{4}$ has been widely used in metal-free (photo)catalysis, ${ }^{11}$ catalyst supports, ${ }^{12}$ sensing, ${ }^{13}$ and bioimaging. ${ }^{14}$ It shows high fluorescence quantum yield, high photostability, and good water solubility. ${ }^{15}$ Various metal ions, including $\mathrm{Hg}^{2+},{ }^{13,16,17} \mathrm{Fe}^{3+},{ }^{18,19} \mathrm{Cr}^{5+},{ }^{20} \mathrm{Cu}^{2+},{ }^{21-23}$ and $\mathrm{Ag}^{+},{ }^{24,25}$ can be detected by $\mathrm{g}-\mathrm{C}_{3} \mathrm{~N}_{4}$ through fluorescence sensing.

Low-dimensional nanomaterials have received significant attention in the past two decades. The superiority of such materials over traditional materials is due to the remarkable surface, optical, and electronic properties. To date, lowdimensional nanomaterials have showed great potential applications in the fields of energy storage and conversion, ${ }^{26}$

Beijing National Laboratory of Molecular Sciences, Key Laboratory of Green Printing, Institute of Chemistry, Chinese Academy of Sciences, Beijing, PR China. E-mail: yanfw@iccas.ac.cn; yuangq@iccas.ac.cn; Fax: +861062559373 catalysis, ${ }^{27}$ engineering materials, ${ }^{28}$ environmental science $^{29}$ and sensors. ${ }^{30}$ The most widely investigated low-dimensional g$\mathrm{C}_{3} \mathrm{~N}_{4}$ nanomaterial is nanosheet. Similar to graphite, bulk g$\mathrm{C}_{3} \mathrm{~N}_{4}$ are stacked arrays of two-dimensional (2D) layers. g- $\mathrm{C}_{3} \mathrm{~N}_{4}$ nanosheets with the thickness of single or several atomic layers can be facially prepared through ultrasonic assistant liquid exfoliation, ${ }^{31}$ thermal oxidation, ${ }^{32}$ chemical exfoliation ${ }^{33}$ and self-assembly. ${ }^{34}$

A few studies have focused on the one-dimensional (1D) g$\mathrm{C}_{3} \mathrm{~N}_{4}$ nanomaterials. The strong $\pi-\pi$ stacking of tri-s-triazine units is beneficial to fabricate $1 \mathrm{D} \mathrm{g}-\mathrm{C}_{3} \mathrm{~N}_{4}$ nanofiber/nanobelt/ nanotube/nanoribbon. Microfibrous $\mathrm{g}-\mathrm{C}_{3} \mathrm{~N}_{4}$ with a mean diameter of about $0.8 \mu \mathrm{m}$ was synthesized on a large scale by a thermal evaporation method via a vapor-solid process. ${ }^{35} \mathrm{~A}$ solvothermal approach was employed to synthesize g- $\mathrm{C}_{3} \mathrm{~N}_{4}$ nanobelts about $50-60 \mathrm{~nm}$ in width by copolymerizing cyanuric chloride and melamine. ${ }^{36}$ Nanotubular $\mathrm{g}-\mathrm{C}_{3} \mathrm{~N}_{4}$ with a diameter of 300-500 nm was prepared by heating precursors synthesized via self-assembly of protonated melamine in glycol. ${ }^{37}$ g- $\mathrm{C}_{3} \mathrm{~N}_{4}$ nanoribbons were synthesized by a one-step hydrothermal method with the existence of graphene oxide. ${ }^{38}$ Although a number of techniques were developed to prepare $1 \mathrm{D} g-\mathrm{C}_{3} \mathrm{~N}_{4}$ nanomaterials, the fabrication of $1 \mathrm{D} g-\mathrm{C}_{3} \mathrm{~N}_{4}$ with small size and large length-diameter ratio via environmentally friendly strategies is still a challenge. ${ }^{18}$

Herein, we report a thermal evaporation combined selfassembly method for the preparation of carbon nitride nanofibers. Self-assembly/gelation of the carbon nitride aqueous solution derived from thermal evaporation of bulk $\mathrm{g}-\mathrm{C}_{3} \mathrm{~N}_{4}$ resulted in carbon nitride nanofibrous hydrogel. Preparation of carbon nitride nanofibrous hydrogel was recently reported through hydrolyzing bulk g- $\mathrm{C}_{3} \mathrm{~N}_{4}$ in $3 \mathrm{M} \mathrm{NaOH}$ aqueous solution. ${ }^{39}$ Our novel method successfully avoids using a large amount of corrosive and harmful reagents. The as-prepared nanofibers with 
diameter of 10-15 $\mathrm{nm}$ and length of $0.5-3 \mu \mathrm{m}$ show excellent dispersion stability in water. Additionally, the $\mathrm{g}-\mathrm{C}_{3} \mathrm{~N}_{4}$ nanofibrous suspension exhibits strong fluorescence and can be employed as a fluorosensor for the selective detection of $\mathrm{Cu}^{2+}$.

\section{Experimental}

\subsection{Reagents and materials}

Melamine was purchased from Sinopharm Chemical Reagent Co., Ltd. $\mathrm{Cu}\left(\mathrm{NO}_{3}\right)_{2}, \mathrm{HgCl}_{2}, \mathrm{AgNO}_{3}, \mathrm{Cr}\left(\mathrm{NO}_{3}\right)_{3}, \mathrm{Fe}\left(\mathrm{NO}_{3}\right)_{3}, \mathrm{Ni}\left(\mathrm{NO}_{3}\right)_{2}$, $\mathrm{Pb}\left(\mathrm{NO}_{3}\right)_{2}, \mathrm{Cd}\left(\mathrm{NO}_{3}\right)_{2}, \mathrm{Mn}\left(\mathrm{NO}_{3}\right)_{2}, \mathrm{NaNO}_{3}, \mathrm{Ca}\left(\mathrm{NO}_{3}\right)_{2}$ and quinine sulphate were purchased from Aladdin Reagent Co., Ltd. All reagents were analytical reagent grade and were used as received without further purification.

\subsection{Synthesis of materials}

Synthesis of bulk $\mathrm{g}-\mathrm{C}_{3} \mathbf{N}_{4}$ (BCN). BCN was synthesized by previously reported method. ${ }^{\mathbf{4}}$ Typically, melamine was directly heated at $550{ }^{\circ} \mathrm{C}$ for $2 \mathrm{~h}$ in still air. The heating rate was $5{ }^{\circ} \mathrm{C}$ $\min ^{-1}$. After cooled to room temperature, BCN was ground to a homogeneously yellow powder for further use or characterization.

Synthesis of carbon nitride nanofibers (CNNF). CNNF was synthesized by self-assembly of the carbon nitride aqueous solution obtained by thermal evaporation of bulk $\mathrm{g}-\mathrm{C}_{3} \mathrm{~N}_{4}$. As demonstrated in Scheme 1, BCN (3.5 g) was pressed into a quartz boat and placed in the central region of a quartz tube $\left(0.5 \mathrm{~m}, 25 \mathrm{~mm}\right.$ ID). The quartz tube was heated to $680{ }^{\circ} \mathrm{C}$ within $1 \mathrm{~h}$ under argon flux (100 $\left.\mathrm{mL} \mathrm{min}{ }^{-1}\right)$, and maintained at this temperature for $2 \mathrm{~h}$. BCN was sublimed at high temperature to form carbon nitride vapor. The vapor was brought out by argon and introduced into a $100 \mathrm{~mL}$ flask containing $50 \mathrm{~mL}$ deionized water. The vapor was dissolved in the deionized water and a brownish yellow aqueous solution was formed. The carbon nitride aqueous solution was centrifuged at $1000 \mathrm{rpm}$ for $5 \mathrm{~min}$ to remove a small amount of solid sample. These solid samples are BCN particles brought out by argon during heating. The supernatant was maintained at room temperature. The color became darker gradually. Finally, a black hydrogel with a concentration of about $0.5 \mathrm{wt} \%$ was formed after $4 \mathrm{~h}$. Dilution of the hydrogel with deionized water, a fluorescent CNNF suspension was obtained. For characterizations, the hydrogel was centrifuged at $10000 \mathrm{rpm}$ for $10 \mathrm{~min}$, and the obtained $\mathrm{CNNF}$ was washed with ethanol and dried at $60{ }^{\circ} \mathrm{C}$ under vacuum over night.

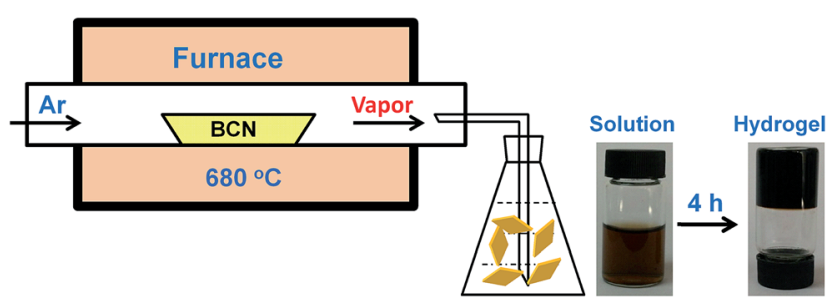

Scheme 1 Illustration of carbon nitride hydrogel formation via selfassembly/gelation of carbon nitride aqueous solution.

\subsection{Characterizations}

Scanning electron microscopy (SEM) images were collected on a Hitachi S-4800 instrument. Transmission electron microscopy (TEM) images were collected on a JEOL JSM-2100 instrument with an accelerating voltage of $200 \mathrm{kV}$. Powder X-ray diffraction (XRD) dates were obtained on a Rigaku Rotaex diffractometer equipped with a $\mathrm{Cu} \mathrm{K} \alpha$ radiation source $(40 \mathrm{kV}, 200 \mathrm{~mA} ; \lambda=$ $1.54056 \AA$ ). Fourier transform infrared (FT-IR) spectra were recorded on a Bruker Tensor 27 by using $\mathrm{KBr}$ pellets. X-ray photoelectron spectroscopy (XPS) data were recorded on a Thermo Scientific ESCALab 250Xi using $200 \mathrm{~W}$ monochromated $\mathrm{Al} \mathrm{K} \alpha$ radiation. Elemental analysis experiments were performed on a Flash EA 1112. Thermogravimetric analyses (TGA) were performed on a Pyris 1 TGA (Perkin Elmer) at a heating rate of $10{ }^{\circ} \mathrm{C} \mathrm{min}^{-1}$ in air flux. UV-visible (UV-vis) absorption spectra were collected on a Shimadzu UV-2600 UVvisible spectrometer. Fluorescence spectra were recorded on a Hitachi F-4500 fluorescence spectrometer.

\subsection{Fluorescence sensing of $\mathrm{Cu}^{2+}$}

Typically, $20 \mu \mathrm{L}$ of CNNF suspension $\left(0.5 \mathrm{mg} \mathrm{mL}^{-1}\right)$ was mixed with $10 \mathrm{~mL}$ of $25 \mathrm{mM}$ phosphate buffer solution ( $\mathrm{pH} 7.0$ ) containing different concentrations of $\mathrm{Cu}^{2+}$ or other metal ions. The fluorescence emission spectra with an excitation wavelength of $335 \mathrm{~nm}$ were recorded at room temperature after $10 \mathrm{~min}$ of reactions.

\section{Results and discussion}

\subsection{Characterization of materials}

It was reported that $\mathrm{g}-\mathrm{C}_{3} \mathrm{~N}_{4}$ obtained by direct heating melamine are bulk morphology with several micrometers in size. ${ }^{\mathbf{4 1}}$ The thermal stability of BCN is so high that the sublimation and pyrolysis only can occur above $600{ }^{\circ} \mathrm{C} .{ }^{42}$ When $\mathrm{BCN}$ was heated at $680{ }^{\circ} \mathrm{C}$ under argon flux, it was sublimed slowly and carbon nitride vapor was generated continuously. A hydrogel was formed via self-assembly/gelation of carbon nitride aqueous solution (Scheme 1). After dilution of the hydrogel with deionized water to $0.5 \mathrm{mg} \mathrm{mL} \mathrm{mL}^{-1}$, a fluorescent suspension can be obtained. This suspension is very stable and no precipitates can be found within several months. SEM images of CNNF in Fig. 1a clearly indicate the formation of a large amount of nanofibers with homogeneous diameters. Most of them are 0.5-3 $\mu \mathrm{m}$ long. TEM images (Fig. 1b) show that the diameters of the nanofibers are in the range of 10-15 $\mathrm{nm}$.

The crystal structures of the samples were characterized by XRD. As presented in Fig. 2, BCN shows two obvious characteristic diffraction peaks. The strong peak located at $27.4^{\circ}$ corresponds to the typical interlayer-stacking structures (002). The weak one located at $13.0^{\circ}$ belongs to the in-planar repeating tri-s-triazine units (100). ${ }^{43}$ CNNF exhibits a similar (002) peak, suggesting the interlayer-stacking structures have not been destroyed. The crystallinity of CNNF decreased slightly, which can be explained by the weakened intensity of the (002) peak. No (100) peak for CNNF was observed. This may be caused by the partial pyrolysis and structural deform of tri-s-triazine units. 

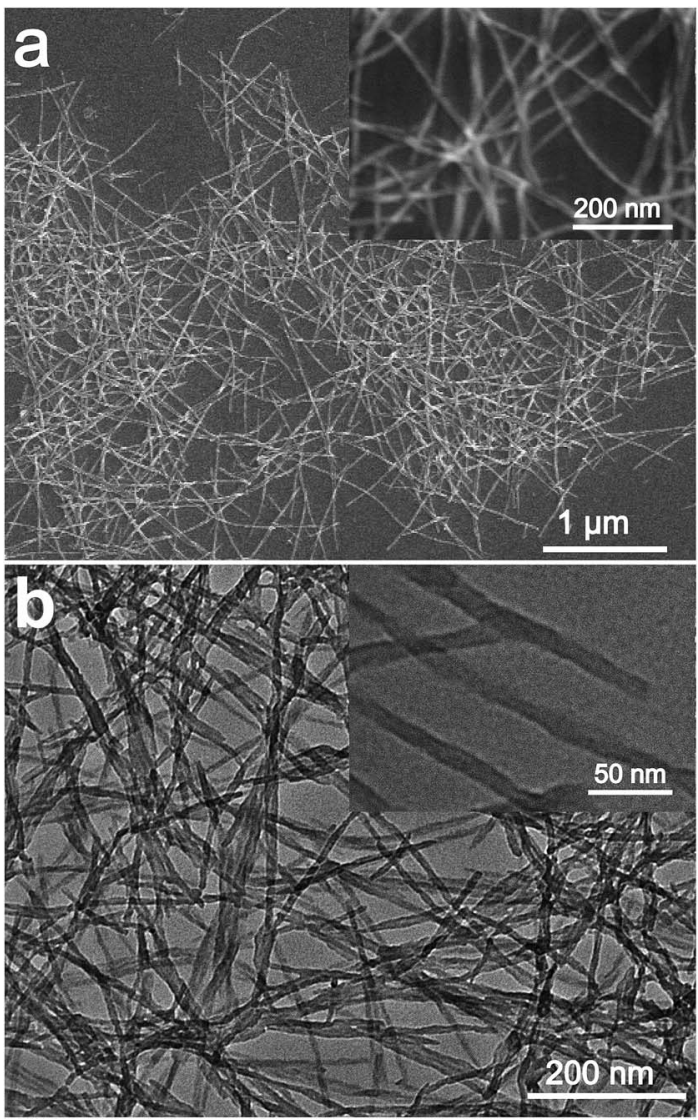

Fig. 1 SEM (a) and TEM (b) images of CNNF.

The pyrolysis of tri-s-triazine units induced an increased interlayer distance, which is identified by the shift of (002) peak from 27.4 to $27.2^{\circ}$.

The partial pyrolysis of tri-s-triazine units was further investigated by FT-IR spectra (Fig. 2b). BCN exhibits a sharp absorption peak at $806 \mathrm{~cm}^{-1}$. This peak can be assigned to the breathing mode of tri-s-triazine units. ${ }^{\mathbf{4 4}}$ As can be seen, it becomes weaker and shows a little shift to $810 \mathrm{~cm}^{-1}$ for CNNF, which results from the partial decomposition of tri-s-triazine units. This is also confirmed by the obviously changed absorption peaks in $1250-1650 \mathrm{~cm}^{-1}$ region. The signal at 1384 $\mathrm{cm}^{-1}$, which belongs to the secondary $(2 \mathrm{C}-\mathrm{N})$ and tertiary $(3 \mathrm{C}-$ $\mathrm{N}$ ) amine fragments, becomes stronger, indicating the breaking of $\mathrm{C}=\mathrm{N}$ and the generation of $\mathrm{C}-\mathrm{N} .{ }^{35}$ During the decomposition of tri-s-triazine units, a small amount of cyano group is generated, which can be identified by the obvious absorption peak at $2226 \mathrm{~cm}^{-1}$. After thermal evaporation, the intensities of the signals corresponding to the $\mathrm{N}-\mathrm{H}$ bond at $3180 \mathrm{~cm}^{-1}$ and the $\mathrm{O}-\mathrm{H}$ bond at $3370 \mathrm{~cm}^{-1}$ are enhanced compared to that of $\mathrm{BCN}$. This indicates that some hydroxyl and amino groups $\left(-\mathrm{NH}_{2}\right.$ or $=\mathrm{NH}$ groups) were formed during thermal evaporation and subsequent self-assembly/gelation in aqueous solution. ${ }^{45}$ Hydroxyl and amino groups provide CNNF with excellent dispersion stability in water.

EA and XPS were employed to investigate the chemical compositions. As shown in Table 1 , the $\mathrm{C} / \mathrm{N}$ atomic ratio of $\mathrm{BCN}$
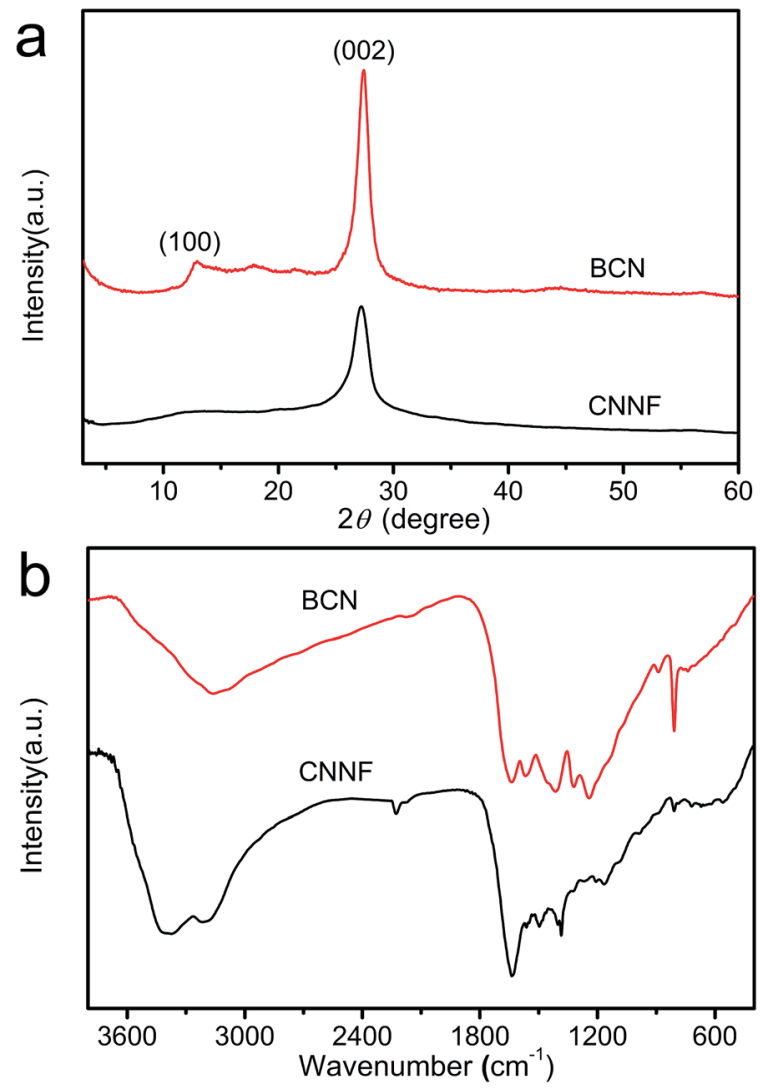

Fig. 2 XRD patterns (a) and FT-IR spectra (b) of BCN and CNNF.

Table 1 EA and XPS results of $B C N$ and $C N N F$

\begin{tabular}{llllrll}
\hline & Samples & $\mathrm{C}^{a}$ & $\mathrm{~N}^{a}$ & $\mathrm{O}^{a}$ & $\mathrm{H}^{a}$ & $\mathrm{C}^{2} \mathrm{~N}^{b}$ \\
\hline EA & BCN & 34.3 & 60.9 & 3.0 & 1.8 & 0.66 \\
& CNNF & 37.0 & 42.9 & 16.8 & 3.3 & 1.00 \\
XPS & BCN & 43.3 & 53.0 & 3.7 & & 0.95 \\
& CNNF & 50.5 & 40.1 & 9.4 & & 1.47
\end{tabular}

${ }^{a}$ wt $\% .{ }^{b}$ Atomic ratio.

determined by EA is 0.66, which is much lower than the theoretical value $(0.75)$. This can be explained by the incomplete polymerization of nitrogen-rich intermediates during condensation. ${ }^{46}$ The $\mathrm{C} / \mathrm{N}$ atomic ratio of $\mathrm{CNNF}$ increases to 1.00 . Clearly, compared to carbon, nitrogen is preferentially lost during thermal evaporation due to the lower thermal stability. XPS measurements present a similar result. As shown in Fig. 3a, both BCN and CNNF are mainly composed of carbon, nitrogen and oxygen elements. The $\mathrm{C} / \mathrm{N}$ atomic ratio is increased largely from 0.95 for $\mathrm{BCN}$ to 1.47 for CNNF. To further clarify the chemical structure of CNNF, the high-resolution of C 1s XPS spectra were investigated (Fig. 3b). The C 1s XPS spectrum of BCN was fitted into two peaks. The peak at a binding energy of $288.1 \mathrm{eV}$ is ascribed to carbon in tri-s-triazine units, and the peak at $284.8 \mathrm{eV}$ is ascribed to graphite carbon. ${ }^{47}$ The content of graphite carbon for $\mathrm{CNNF}$ is comparable to that of $\mathrm{BCN}$. 

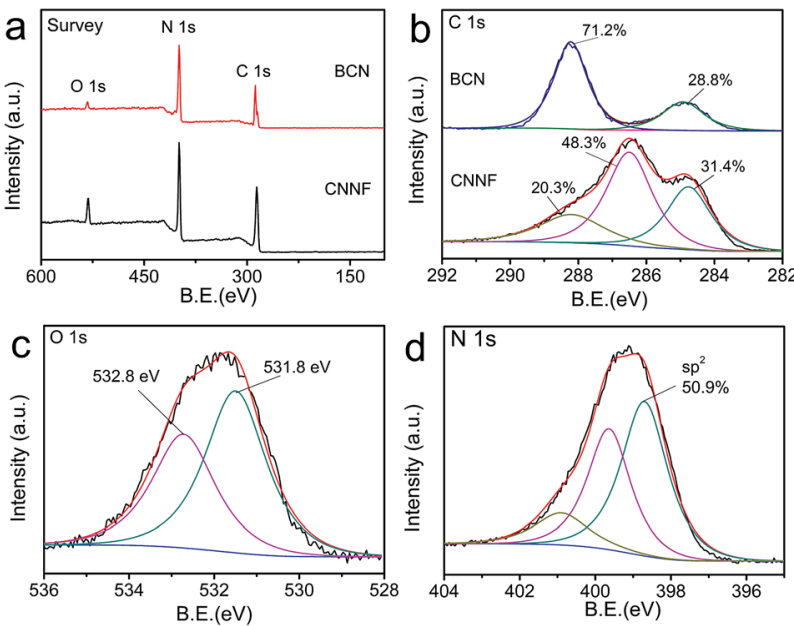

Fig. 3 XPS survey spectra (a) and C 1s XPS spectra (b) of BCN and CNNF. O 1s XPS spectrum (c) and N 1s XPS spectrum (d) of CNNF.

However, the carbon in tri-s-triazine units decreased sharply from $71.2 \%$ for BCN to $20.3 \%$ for CNNF. A new chemical state of carbon at $286.5 \mathrm{eV}$ was found in the C 1s XPS spectrum of CNNF. This can be attributed to $\mathrm{C}-\mathrm{N}$ and $\mathrm{C}-\mathrm{O}$ species, which is accordance with the FT-IR results. ${ }^{48}$ The existence of $\mathrm{C}-\mathrm{OH}$ species was also confirmed by O 1s XPS spectrum. As shown in Fig. 3c, the $\mathrm{O}$ 1s XPS spectrum was fitted into two peaks at 532.8 and $531.8 \mathrm{eV}$. These signals could be ascribed to the adsorbed water and $\mathrm{C}-\mathrm{OH}$ species respectively. ${ }^{49}$

Due to the high thermal stability, tri-s-triazine units cannot be decomposed completely. This was already demonstrated by the FT-IR and C 1s XPS measurements. To further clarify the chemical states of nitrogen atoms, N 1s XPS spectrum was fitted into three peaks with the binding energies of 398.7, 399.6 and $400.9 \mathrm{eV}$, corresponding to the $\mathrm{sp}^{2}$-hybridized nitrogen within aromatic rings, tertiary nitrogen connected with aromatic rings and terminal amino groups, respectively. ${ }^{50}$ The content of the $\mathrm{sp}^{2}$ nitrogen is $50.9 \%$ (Fig. 3d), suggesting that about half of the nitrogen atoms exist in the form of tri-s-triazine or $s$-triazine. These nitrogen containing aromatic rings provide CNNF with $\pi-\pi$ stacked structures and specific optical properties.

The thermal behaviour of CNNF was measured by TGA analysis under air flux. For comparison, the result for BCN was also presented. As shown in Fig. 4, BCN shows an obvious

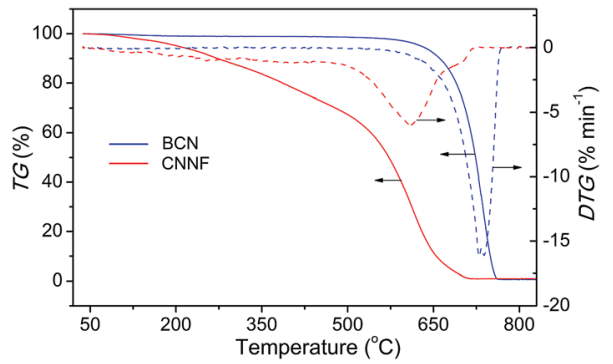

Fig. 4 TGA curves of $B C N$ and $C N N F$ at a heating rate of $10^{\circ} \mathrm{C} \mathrm{min}^{-1}$ under air flux. weight loss above $600{ }^{\circ} \mathrm{C}$, related to the sublimation and pyrolysis of the tri-s-triazine units. CNNF shows a markedly different thermal behavior. The TGA curve exhibits a moderate weight loss between 100 and $500{ }^{\circ} \mathrm{C}$ due to the loss of amino, hydroxyl and $s$-triazine groups. Above $500{ }^{\circ} \mathrm{C}$, the weight loss accelerates, which is ascribed to the polymerization of $s$-triazine units and pyrolysis of tri-s-triazine units.

\subsection{Mechanism for the formation of nanofibers and hydrogels}

In order to investigate the mechanism for the formation of nanofibers and hydrogels, time-dependent TEM images of the self-assembled products were provided in Fig. 5. Nanoparticles with 10-15 $\mathrm{nm}$ in diameters and a small amount of short nanorods with the same diameters are found in the immediately obtained carbon nitride aqueous solution (Scheme 1). After maintained at room temperature for $0.5 \mathrm{~h}$, the colour of the solution becomes darker gradually. The nanoparticles are disappeared and nanorods with lengths of tens to hundreds of nanometers are obtained. The lengths of the nanorods increase continuously with the increase in time. Finally, nanofibers with the lengths of $0.5-3 \mu \mathrm{m}$ are formed after $4 \mathrm{~h}$. At the same time, the viscosity of the solution increases continuously, a black hydrogel is obtained (Scheme 1). These observations as well as the conclusions made from the above analyses of the crystal structures and chemical compositions allow proposing a mechanism for the formation of nanofibers and hydrogels. Firstly, BCN is sublimed at $680^{\circ} \mathrm{C}$ to form carbon nitride vapor. Some amino groups are generated during the partial decomposition. Carbon nitride vapor is then dissolved in water and carbon nitride aqueous solution is obtained. Simultaneously, some carbon nitride moleculars react with water to form
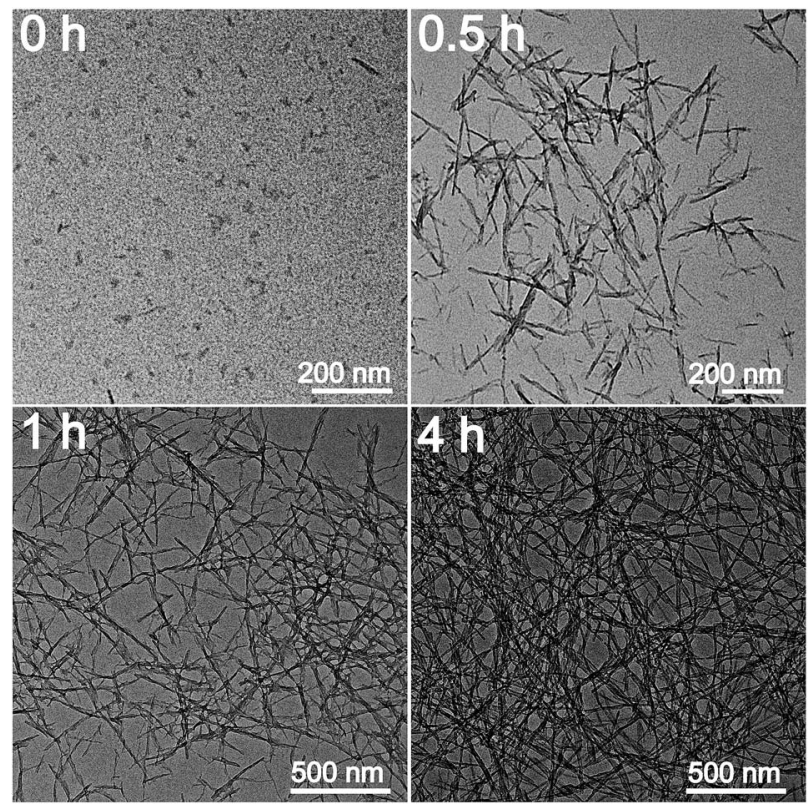

Fig. 5 TEM images of the self-assembled products obtained at different times. 
hydroxyl groups. The existence of amino and hydroxyl groups means that extensive hydrogen bonding may take place, which leads to the self-assembly and generation of nanoparticles. Due to the high thermal stability of BCN, some aromatic nucleuses in the form of $s$-triazine or tri-s-triazine are left in the carbon nitride moleculars. The $\pi-\pi$ stacking of these aromatic nucleuses as well as the hydrogen bonding serves as the driving force for the further growth of nanoparticles to nanofibers. ${ }^{51}$ Finally, hydrogel is formed via self-organization of the obtained fibrous materials. $^{52}$

\subsection{Optical properties of CNNF suspension}

The optical properties of CNNF suspensions were investigated by UV-vis absorption spectrum and fluorescence excitation and emission spectra. As shown in Fig. 6a, BCN gives a strong UV-vis absorption with an absorption edge of about $450 \mathrm{~nm}$. Due to the partial decomposition of tri-s-triazine units, CNNF demonstrates a different UV-vis absorption. A absorption peak cantered at around $295 \mathrm{~nm}$ is observed, which may be ascribed to the $\pi-\pi^{*}$ electronic transitions. ${ }^{53}$ The terminal groups provide CNNF with an obvious absorption shoulder between 400 and $600 \mathrm{~nm} .{ }^{54}$ Strong fluorescence excitation peak is presented at around $335 \mathrm{~nm}$ (Fig. 6b). Under the excitation wavelength of $335 \mathrm{~nm}$, CNNF exhibits an emission peak cantered at around $435 \mathrm{~nm}$. Excitation wavelength affects the fluorescence emission largely (Fig. 6c). When the excitation wavelength increases from 280 to $320 \mathrm{~nm}$, the emission peak of CNNF shows no obvious shift but the fluorescence intensity increases significantly. Further increase in the excitation wavelengths, the emission peak shows an obvious red shift, and the intensity decreases gradually. The excitation-dependent emission feature indicates that CNNF contains more than one emitter. This can be ascribed to the abundant surface functional groups in CNNF. $^{19}$ The relative fluorescence quantum yield of CNNF
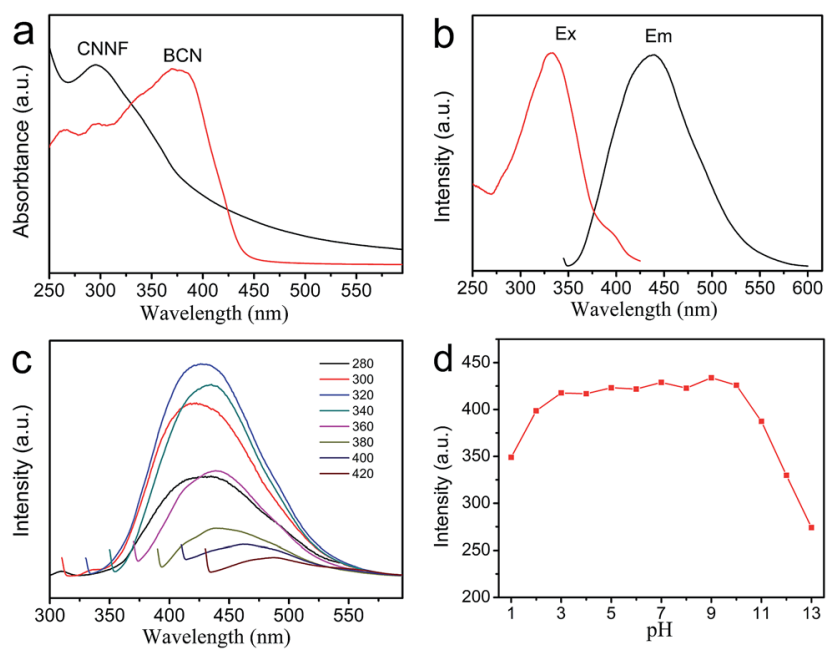

Fig. 6 (a) UV-vis absorption spectra of BCN and CNNF suspension. (b) Fluorescence excitation and emission spectra of CNNF suspension. (c) Fluorescence emission spectra of CNNF suspension corresponding to different excitation wavelengths from $280-420 \mathrm{~nm}$. (d) Effect of $\mathrm{pH}$ on the fluorescence intensity of CNNF suspension ( $\lambda_{\mathrm{ex}}=335 \mathrm{~nm}$ ).
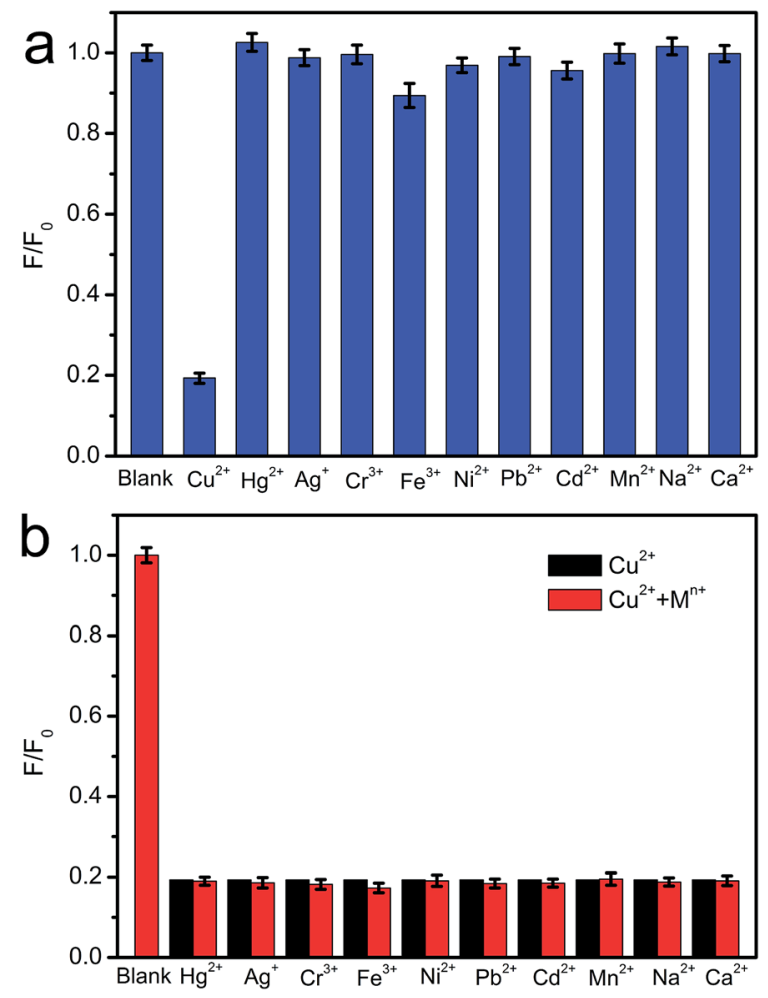

Fig. 7 (a) Fluorescence selectivity of CNNF suspension in the presence of $50 \mu \mathrm{M}$ of various metal ions. (b) Fluorescence response of CNNF suspension towards $50 \mu \mathrm{M} \mathrm{Cu}^{2+}$ and interference of $50 \mu \mathrm{M}$ of other metal ions with $50 \mu \mathrm{M} \mathrm{Cu}{ }^{2+}$.

suspension was determined to be $7.26 \%$ in reference to quinine sulphate. The effect of the solution $\mathrm{pH}$ on the fluorescence intensity of the CNNF suspension was also investigated. It can be seen from Fig. 6d that no obvious fluctuation in the fluorescence intensity was observed within the $\mathrm{pH}$ range from 3 to 10 .

\subsection{Fluorescence sensing of $\mathrm{Cu}^{2+}$}

The excellent fluorescence properties make CNNF a potential fluorescent sensor for detection of metal ion. As shown in Fig. 7a, fluorescence responses of CNNF are different in the presence of different metal ions $(50 \mu \mathrm{M})$. The fluorescence intensity was greatly quenched by adding $\mathrm{Cu}^{2+}$. It is reported that the fluorescence responses of $\mathrm{g}-\mathrm{C}_{3} \mathrm{~N}_{4}$ based nanomaterials is sensitive to $\mathrm{Hg}^{2+},{ }^{55} \mathrm{Ag}^{+},{ }^{24}$ and $\mathrm{Fe}^{3+} .{ }^{18}$ For CNNF, these ions as well as other ions $\left(\mathrm{Cr}^{3+}, \mathrm{Ni}^{2+}, \mathrm{Pb}^{2+}, \mathrm{Cd}^{2+}, \mathrm{Mn}^{2+}, \mathrm{Na}^{+}\right.$and $\left.\mathrm{Ca}^{2+}\right)$ tested under the same conditions show no obvious effects on the fluorescence intensities. The interference of these metal ions was further investigated. As shown in Fig. 7b, no significant differences of the fluorescence intensities between CNNF + $\mathrm{Cu}^{2+}$ and $\mathrm{CNNF}+\mathrm{Cu}^{2+}+\mathrm{M}^{n+}$ systems are observed, indicating that $\mathrm{CNNF}$ is an effective sensing material for the selective detection of $\mathrm{Cu}^{2+}$.

The quenching mechanism was investigated by XPS spectrum. Fig. 8 shows the $\mathrm{Cu} 2 \mathrm{p}_{3 / 2}$ spectrum of CNNF treated with $\mathrm{Cu}^{2+}$. The spectrum is fitted into two peaks. One at $932.8 \mathrm{eV}$ is 


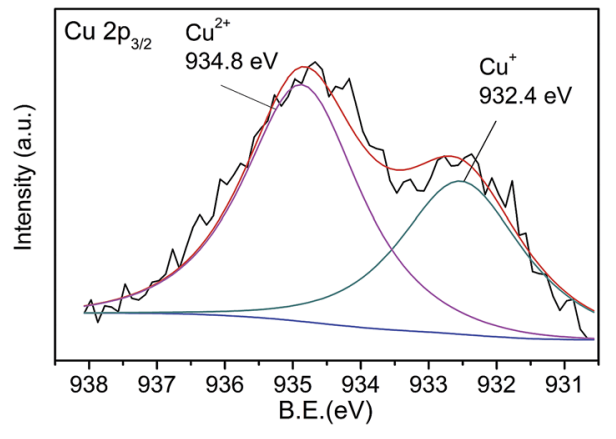

Fig. $8 \mathrm{Cu} 2 \mathrm{p}_{3 / 2}$ XPS spectrum of CNNF treated with $\mathrm{Cu}^{2+}$.

derived from $\mathrm{Cu}^{2+}$. The other at $934.8 \mathrm{eV}$ is ascribed to $\mathrm{Cu}^{+}$. The $\mathrm{Cu}^{+}$species are stemmed from the reduction of $\mathrm{Cu}^{2+}$. The redox potential of $\mathrm{Cu}^{2+} / \mathrm{Cu}^{+}$lies between the conduction band (CB) and valence band (VB) of CNNF. The photoinduced electron is transferred from the $\mathrm{CB}$ of $\mathrm{CNNF}$ to $\mathrm{Cu}^{2+}$, which leads to the reduction of $\mathrm{Cu}^{2+}$ to $\mathrm{Cu}^{+}$. Meanwhile, the fluorescence of $\mathrm{CNNF}$ is quenched..$^{24}$

The dependence of fluorescence intensity on the concentration of $\mathrm{Cu}^{2+}$ was studied. As shown in Fig. 9a, the fluorescence intensity decreases gradually with the increase in $\mathrm{Cu}^{2+}$ concentration. Two good linearities between the fluorescence intensity and the $\mathrm{Cu}^{2+}$ concentration are observed in $0-0.8 \mu \mathrm{M}$
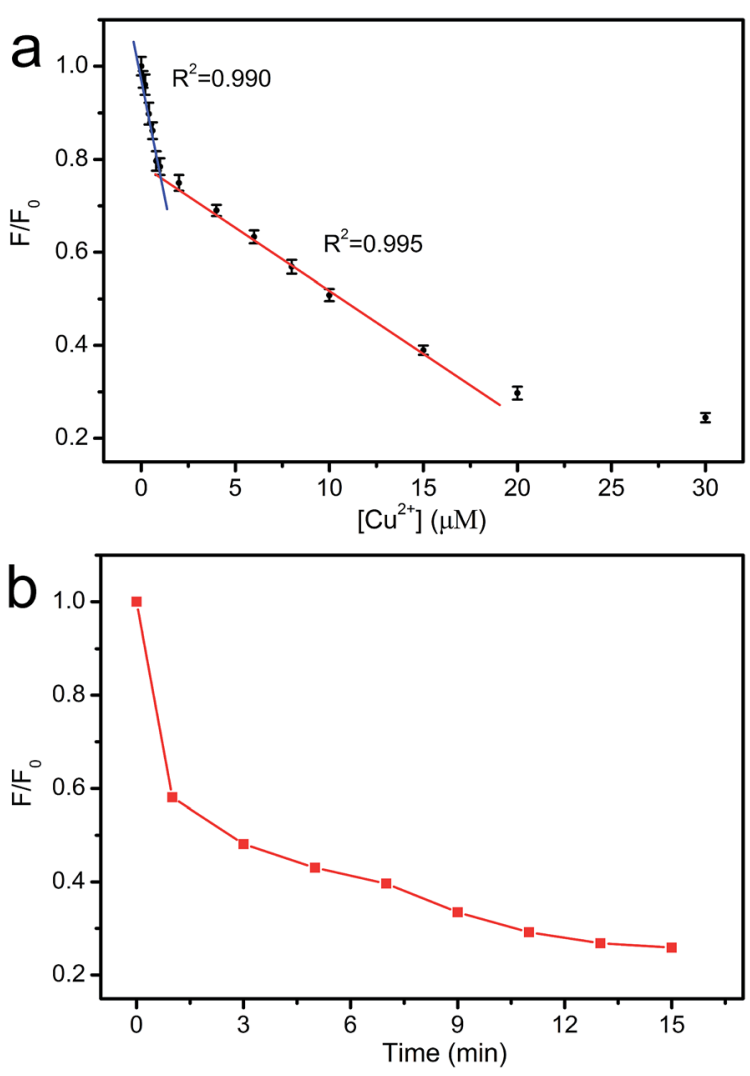

Fig. 9 (a) The relationship between $F / F_{0}$ and the concentration of $\mathrm{Cu}^{2+}$. (b) Time-dependent fluorescence intensity of CNNF suspension $\left(\lambda_{\mathrm{ex}}=335 \mathrm{~nm}\right)$.

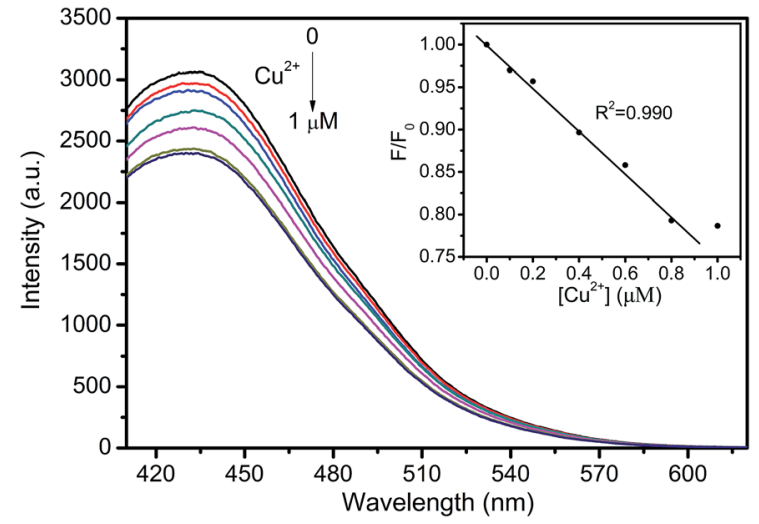

Fig. 10 Fluorescence spectra of CNNF dispersion in the presence of different $\mathrm{Cu}^{2+}$ concentrations $(0,0.1,0.2,0.4,0.6,0.8$ and $1 \mu \mathrm{M})$ in tap water. Inset: the relationship between $F / F_{0}$ and the concentration of $\mathrm{Cu}^{2+}$.

and $0.8-15 \mu \mathrm{M}$ region, respectively. Two different interaction sites for $\mathrm{Cu}^{2+}$ may exist in CNNF. ${ }^{16}$ At the concentration of $0-0.8$ $\mu \mathrm{M}, \mathrm{Cu}^{2+}$ interacts preferentially with the stronger sites. At the concentration of $0.8-15 \mu \mathrm{M}, \mathrm{Cu}^{2+}$ interacts with the weaker sites. The time-dependent fluorescence spectra of the CNNF suspension treated with $\mathrm{Cu}^{2+}$ in Fig. 9b indicate a similar result. The fluorescence intensity quenches quickly within the first 1 minute, which is ascribed to the interaction of $\mathrm{Cu}^{2+}$ with the stronger sites. While the stronger sites are depleted, $\mathrm{Cu}^{2+}$ interacts with the weaker sites, leading to a slower fluorescence quenching. The content of the stronger sites is lower than that of the weaker sites, which can be explained by the relatively narrow concentration range of $\mathrm{Cu}^{2+}$ for the first linearity. The detection limit was calculated to be $0.06 \mu \mathrm{M}$.

Tap water was selected as the real sample to investigate the practical application of the fluorescent probe (Fig. 10). CNNF exhibits excellent selectivity to $\mathrm{Cu}^{2+}$. Other metal ions in tap water have little effect on the fluorescence intensities of CNNF. Adding $\mathrm{Cu}^{2+}$ into the tap water led to a significant fluorescence quenching. The fluorescence intensity decreases with the increase of $\mathrm{Cu}^{2+}$ concentration from 0 to $1 \mu \mathrm{M}$. Good linear relationship between the fluorescence intensity and the $\mathrm{Cu}^{2+}$ concentration can be observed in the range of $0-0.8 \mu \mathrm{M}$. This implies that the fluorescent probe has potential application in real water samples for the detection of $\mathrm{Cu}^{2+}$.

\section{Conclusions}

In conclusion, we reported a fluorescent carbon nitride nanofibrous hydrogel for the selective sensing of $\mathrm{Cu}^{2+}$. Thermal evaporation and subsequent self-assembly/gelation was proved to be an effective method for the preparation of carbon nitride nanofibrous hydrogel. The $\pi-\pi$ stacking of the nitrogen containing aromatic rings and the hydrogen bonding serve as the driving forces for the formation of nanofibrous hydrogel. The fluorescent nanofiber suspension was obtained by dilution of the hydrogel with water. The excellent fluorescence properties and the high dispersion stability in water make carbon nitride 
nanofiber promising for the sensing application in the selective detection of $\mathrm{Cu}^{2+}$. Therefore, our present study has opened a facile way for the synthesis of $1 \mathrm{D} g-\mathrm{C}_{3} \mathrm{~N}_{4}$ nanomaterials with improved structural and optical properties.

\section{Acknowledgements}

This work was supported by the National Natural Science Foundation of China (21304101).

\section{Notes and references}

1 L. Song, Z. Wu, H. Liang, F. Zhou, Z. Yu, L. Xu, Z. Pan and S. Yu, Nano Energy, 2016, 19, 117-127.

2 Q. Wei, X. Tong, G. Zhang, J. Qiao, Q. Gong and S. Sun, Catalysts, 2015, 5, 1574-1602.

3 Y. Deng, Y. Xie, K. Zou and X. Ji, J. Mater. Chem. A, 2016, 4, 1144-1173.

4 J. P. Paraknowitsch and A. Thomas, Energy Environ. Sci., 2013, 6, 2839-2855.

5 Y. Wang, X. Wang and M. Antonietti, Angew. Chem., Int. Ed., 2012, 51, 68-89.

6 Y. Zheng, J. Liu, J. Liang, M. Jaroniec and S. Z. Qiao, Energy Environ. Sci., 2012, 5, 6717-6731.

7 X. Wang, K. Maeda, A. Thomas, K. Takanabe, G. Xin, J. M. Carlsson, K. Domen and M. Antonietti, Nat. Mater., 2009, 8, 76-80.

8 Y. Gong, M. Li, H. Li and Y. Wang, Green Chem., 2015, 17, 715-736.

9 F. Dong, L. Wu, Y. Sun, M. Fu, Z. Wu and S. C. Lee, J. Mater. Chem., 2011, 21, 15171-15174.

10 Z. Huang, F. Li, B. Chen and G. Yuan, Catal. Sci. Technol., 2014, 4, 4258-4264.

11 J. Zhu, P. Xiao, H. Li and S. A. Carabineiro, ACS Appl. Mater. Interfaces, 2014, 6, 16449-16465.

12 Z. Huang, F. Li, B. Chen, F. Xue, G. Chen and G. Yuan, Appl. Catal., A, 2011, 403, 104-111.

13 H. Zhang, Y. Huang, S. Hu, Q. Huang, C. Wei, W. Zhang, L. Kang, Z. Huang and A. Hao, J. Mater. Chem. C, 2015, 3, 2093-2100.

14 X. Zhang, X. Xie, H. Wang, J. Zhang, B. Pan and Y. Xie, J. Am. Chem. Soc., 2013, 135, 18-21.

15 Z. X. Zhou, Y. F. Shen, Y. Li, A. R. Liu, S. Q. Liu and Y. J. Zhang, ACS Nano, 2015, 9, 12480-12487.

16 J. Ma, B. Guo, X. Cao, Y. Lin, B. Yao, F. Li, W. Weng and L. Huang, Talanta, 2015, 143, 205-211.

17 X. Cao, J. Ma, Y. Lin, B. Yao, F. Li, W. Weng and X. Lin, Spectrochim. Acta, Part A, 2015, 151, 875-880.

18 J. Tian, Q. Liu, A. M. Asiri, X. Sun and Y. He, Sens. Actuators, $B, 2015,216,453-460$.

19 S. Zhang, J. Li, M. Zeng, J. Xu, X. Wang and W. Hu, Nanoscale, 2014, 6, 4157-4162.

20 M. Rong, L. Lin, X. Song, Y. Wang, Y. Zhong, J. Yan, Y. Feng, X. Zeng and X. Chen, Biosens. Bioelectron., 2015, 68, 210-217.

21 M. Rong, X. Song, T. Zhao, Q. Yao, Y. Wang and X. Chen, J. Mater. Chem. C, 2015, 3, 10916-10924.
22 J. Tian, Q. Liu, A. M. Asiri, A. O. Al-Youbi and X. Sun, Anal. Chem., 2013, 85, 5595-5599.

23 B. Xia, M. Chu, S. Wang, W. Wang, S. Yang, C. Liu and S. Luo, Anal. Chim. Acta, 2015, 891, 113-119.

24 H. Huang, R. Chen, J. Ma, L. Yan, Y. Zhao, Y. Wang, W. Zhang, J. Fan and X. Chen, Chem. Commun., 2014, 50, 15415-15418.

25 Y. Cao, W. Wu, S. Wang, H. Peng, X. Hu and Y. Yu, J. Fluoresc., 2016, 26, 739-744.

26 P. Kumar, F. Y. Wu, L. H. Hu, S. Ali Abbas, J. Ming, C. N. Lin, J. Fang, C. W. Chu and L. J. Li, Nanoscale, 2015, 7, 8093-8100.

27 X. L. Pan and X. H. Bao, Acc. Chem. Res., 2011, 44, 553-562.

28 X. Sun, H. Sun, H. Li and H. Peng, Adv. Mater., 2013, 25, 5153-5176.

29 Y. Jin, S. C. Hawkins, C. P. Huynh and S. Su, Energy Environ. Sci., 2013, 6, 2591-2596.

30 Y. Du and S. Guo, Nanoscale, 2016, 8, 2532-2543.

31 S. Yang, Y. Gong, J. Zhang, L. Zhan, L. Ma, Z. Fang, R. Vajtai, X. Wang and P. M. Ajayan, Adv. Mater., 2013, 25, 2452-2456.

32 P. Niu, L. Zhang, G. Liu and H. M. Cheng, Adv. Funct. Mater., 2012, 22, 4763-4770.

33 J. Xu, L. Zhang, R. Shi and Y. Zhu, J. Mater. Chem. A, 2013, 1, 14766-14772.

34 Z. J. Huang, F. B. Li, B. F. Chen and G. Q. Yuan, ChemSusChem, 2016, 9, 478-484.

35 Y. Zhao, Z. Liu, W. Chu, L. Song, Z. Zhang, D. Yu, Y. Tian, S. Xie and L. Sun, Adv. Mater., 2008, 20, 1777-1781.

36 Y. Cui, Z. Ding, X. Fu and X. Wang, Angew. Chem., Int. Ed., 2012, 51, 11814-11818.

37 J. Gao, Y. Zhou, Z. Li, S. Yan, N. Wang and Z. Zou, Nanoscale, 2012, 4, 3687-3692.

38 Y. Zhao, F. Zhao, X. Wang, C. Xu, Z. Zhang, G. Shi and L. Qu, Angew. Chem., Int. Ed., 2014, 53, 13934-13939.

39 Y. Zhang, Z. Zhou, Y. Shen, Q. Zhou, J. Wang, A. Liu, S. Liu and Y. Zhang, ACS Nano, 2016, 10, 9036-9043.

40 Z. Huang, F. Li, B. Chen and G. Yuan, RSC Adv., 2015, 5, 102700-102706.

41 Z. Huang, F. Li, B. Chen and G. Yuan, RSC Adv., 2015, 5, 14027-14033.

42 Z. Yang, Y. Zhang and Z. Schnepp, J. Mater. Chem. A, 2015, 3, 14081-14092.

43 J. Qin, J. Huo, P. Zhang, J. Zeng, T. Wang and H. Zeng, Nanoscale, 2016, 8, 2249-2259.

44 P. Yang, J. Zhao, W. Qiao, L. Li and Z. Zhu, Nanoscale, 2015, 7, 18887-18890.

45 Z. Huang, F. Li, B. Chen and G. Yuan, Catal. Sci. Technol., 2016, 6, 2942-2948.

46 V. W. Lau, M. B. Mesch, V. Duppel, V. Blum, J. Senker and B. V. Lotsch, J. Am. Chem. Soc., 2015, 137, 1064-1072.

47 Z. Zhou, J. Wang, J. Yu, Y. Shen, Y. Li, A. Liu, S. Liu and Y. Zhang, J. Am. Chem. Soc., 2015, 137, 2179-2182.

48 J. Li, B. Shen, Z. Hong, B. Lin, B. Gao and Y. Chen, Chem. Commun., 2012, 48, 12017-12019.

49 G. Dong, Z. Ai and L. Zhang, RSC Adv., 2014, 4, 5553-5560. 50 G. Algara-Siller, N. Severin, S. Y. Chong, T. Bjorkman, R. G. Palgrave, A. Laybourn, M. Antonietti, Y. Z. Khimyak, A. V. Krasheninnikov, J. P. Rabe, U. Kaiser, A. I. Cooper, 
A. Thomas and M. J. Bojdys, Angew. Chem., Int. Ed., 2014, 53, $7450-7455$.

51 M. L. Ma, Y. Kuang, Y. Gao, Y. Zhang, P. Gao and B. Xu, J. Am. Chem. Soc., 2010, 132, 2719-2728.

52 Y. Chen, M. Quan, H. Yu, L. Zhang, H. Yang and Y. Lu, RSC Adv., 2015, 5, 31219-31225.
53 Z. Geng, H. Zhang, Q. Xiong, Y. Zhang, H. Zhao and G. Wang, J. Mater. Chem. A, 2015, 3, 19455-19460.

54 P. Wu, J. Wang, J. Zhao, L. Guo and F. E. Osterloh, J. Mater. Chem. A, 2014, 2, 20338-20344.

55 Y. Lu, J. Chen, A. Wang, N. Bao, J. Feng, W. Wang and L. Shao, J. Mater. Chem. C, 2015, 3, 73-78. 